\title{
Estrategias lúdicas para el favorecimiento de la competencia gramatical del tiempo presente simple del inglés para estudiantes de pregrado
}

\author{
María de Jesús Suárez-Vera*
}

* M.Sc. en Educación. M.Sc. en Tecnología Educativa. Docente, Instituto de Lenguas, Universidad Autónoma de Bucaramanga, Colombia.

Correo electrónico: msuarez@unab.edu.co

Recibido: 21 de octubre del 2013 Aprobado: 12 de noviembre del 2013

Cómo citar este artículo: Suárez-Vera, María de Jesús. "Estrategias lúdicas para el favorecimiento de la competencia gramatical del tiempo presente simple del inglés para estudiantes de pregrado". Rastros Rostros 15.29 (2013): 47-58. Impreso.

\section{Resumen}

Este artículo presenta los resultados de un proyecto de investigación, utilizando la plataforma TEMA-Moodle UNAB para el aprendizaje de la gramática del tiempo presente simple del inglés. Se diseñaron diferentes actividades utilizando el Task-based Learning Approach (TBL) y se tuvo en cuenta, además, una metodología lúdica. El estudio buscaba averiguar cuál es el nivel que alcanzan los estudiantes de pregrado del nivel A1, del programa de inglés de la Universidad Autónoma de Bucaramanga, UNAB, luego de la implementación con la metodología lúdica aplicada para el desarrollo de la competencia gramatical del tiempo presente simple. Para el análisis de los datos se utilizó la media, la desviación estándar y la prueba t-student. La muestra estuvo conformada por un grupo piloto, uno de control y uno experimental, asignados aleatoriamente. Se encontró que los resultados en el postest fueron más altos en el grupo experimental, con un nivel de significancia de 0,05. La mejora en la gramática se debe a las estrategias elegidas, a la manera particular como se diseñó y organizó el material, a la instrucción directa proporcionada durante el taller y a otras características particulares del proyecto de investigación.

Palabras clave: gramática, instrucción directa, metodología lúdica, presente simple.

\section{Recreational Strategies for Improving English Grammar Skills in the Present Simple Tense for Undergraduate Students}

\begin{abstract}
This article presents the results of a research project using the TEMA-Moodle UNAB system to teach simple present tense grammar in English. Different activities were designed following a task-based approach (TBL) and the use of recreational strategies was also taken into account. The study sought to determine the level reached by A1-level undergraduate students at Universidad Autónoma de Bucaramanga (UNAB), after implementing an applied recreational methodology to develop grammar skills in present simple tense. To analyze the data, the median, standard deviation and student's $t$-test were used. The sample consisted of a pilot group, a control group, and an experimental group, assigned randomly. It was found that posttest results were highest in the experimental group, with a significance level of 0.05 . The improvement in grammar was due to the strategies selected, the specific way in which material was designed and organized, the direct instruction provided during the workshop, and other characteristics specific to the research project.
\end{abstract}

Keywords: grammar, direct instruction, recreational methodology, present simple.

Estratégias lúdicas para o favorecimento da competência gramatical do tempo presente simples do inglês, para estudantes de graduação

Resumo

Este artigo apresenta os resultados de um projeto de pesquisa que utiliza a plataforma TEMA-MoodleunAB para a aprendizagem do tempo presente simples do inglês. Foram traçadas diferentes atividades que utilizavam o Task-based Learning Approach (TBL) e foi usada também uma metodologia lúdica. O estudo procurava averiguar qual é o nível que atingem os estudantes de graduação do nível A1 do programa de inglês da Universidade Autônoma de Bucaramanga (UNAB), após a implantação com metodologia lúdica aplicada ao desenvolvimento da competência gramatical do tempo presente simples. Para a análise dos dados, foram utilizados a média, o desvio padrão e a forma t-student. A amostra foi formada por um grupo-piloto, um de controle e outro experimental, formados aleatoriamente. Observou-se que os resultados no pós-teste foram mais altos no grupo experimental com um nível significativo de 00,5 . A melhoria na gramática devese às estratégias escolhidas, à maneira particular que se traçou e organizou o material, à instrução direta proporcionada durante a oficina e a outras características particulares do projeto de pesquisa.

Palavras-chave: gramática, instrução direta, metodologia lúdica, presente simples. 


\section{Introducción}

La presente investigación buscaba aportar soluciones a la problemática del bajo aprendizaje de la gramática del idioma inglés, presentando una metodología lúdica con la que el estudiante lograra desarrollar procesos de aprendizaje significativos y duraderos con su participación activa. Este artículo presenta los resultados del diseño y la aplicación de un taller de 10 horas para la enseñanza-aprendizaje del tiempo presente simple del inglés con el tema daily life, en el que se proponen determinadas estrategias bajo una metodología lúdica apoyada en el enfoque Task-Based Learning, твL, de Willis. Un componente importante para el desarrollo del taller fue el uso de herramientas Web 2.0 apoyadas en la plataforma TEMA-Moodle-UNAB, en la que los estudiantes desarrollaron la totalidad de las tareas.

Con el experimento llevado a cabo se buscaba comprobar la hipótesis sobre la relación existente entre el uso de la metodología lúdica y el desarrollo de la competencia gramatical del tiempo presente simple del inglés. Por medio de la prueba estadística $t$-student se logró comprobar la hipótesis de la investigación planteada, dando como resultado diferencias estadísticamente significativas entre calificaciones entre los grupos experimental y de control, para el pretest y el postest. Aspectos como el marco teórico, el método y los resultados se amplían a continuación.

\section{Marco teórico}

\section{La gramática desde el enfoque comunicativo}

El actual proyecto de investigación se sustenta en los lineamientos dados por el Marco Común Europeo, MCE (Common European Framework of Reference for Languages), que da pautas claras sobre lo que se espera o se exige que los alumnos desarrollen en su competencia gramatical en el nivel A1. Estos aspectos se detallan a continuación:

Para el nivel A1, el MCE establece la escala global de competencia definiendo claramente lo que el aprendiz debe ser capaz de realizar con el idioma:

Es capaz de comprender y utilizar expresiones cotidianas de uso muy frecuente, así como frases sencillas destinadas a satisfacer necesidades de tipo inmediato. Puede presentarse a sí mismo y a otros, pedir y dar información personal básica sobre el lugar donde vive, sus pertenencias y las personas que conoce. Puede relacionarse de forma elemental siempre que su interlocutor hable despacio y con claridad y esté dispuesto a cooperar. (24)

Respecto a los logros, y debido a que la gramática es usada para la comunicación, el MCE describe lo que se espera que el estudiante de este nivel produzca:

Muestra un control limitado de unas pocas estructuras gramaticales sencillas y de modelos de oraciones dentro de un repertorio memorizado. Plantea y contesta preguntas relativas a datos personales. Puede responder y hacer preguntas sobre detalles personales. La comunicación depende totalmente de la repetición y la reparación de frases. Es capaz de enlazar palabras o grupos de palabras con conectores muy básicos y lineales como "y" y “entonces". (29)

Además, el MCE también da pautas claras sobre el tipo de actividades que los alumnos deben realizar para que desarrollen su competencia gramatical:

Si se utilizan ejercicios, se pueden utilizar algunos de los siguientes o todos ellos:

a) rellenar espacios en blanco;

b) construir oraciones con un modelo dado;

c) opciones;

d) ejercicios de sustitución de categorías gramaticales (por ejemplo: singular/plural, presente/pasado, activa/pasiva, etc.); e) relacionar oraciones (por ejemplo: oraciones subordinadas relativas, causales, concesivas, etcétera);

f) traducir oraciones de L1 a L2;

g) ejercicios de pregunta-respuesta utilizando estructuras concretas;

h) ejercicios de fluidez centrados en la gramática. (152)

Este proyecto siguió los lineamientos anteriores dados por el MCE para desarrollar la competencia gramatical del tiempo presente simple del inglés del nivel A1, enfatizando su aprendizaje-enseñanza bajo un enfoque comunicativo en el que el estudiante usa la gramática como un elemento para la comunicación en situaciones de la vida cotidiana. Para este caso se seleccionó la situación comunicativa daily life.

El MCE (109) propone mediante el TBL una serie de tareas comunicativas en la que la gramática se utiliza en transacciones prácticas cotidianas y en situaciones muy limitadas y concretas, de carácter previsible: 
Incluyen, por ejemplo, realizar compras de necesidad muy inmediata o hacer las preguntas básicas para ubicarse en el espacio urbano. En estos casos, se espera que el alumno utilice un repertorio muy limitado de frases, ensayado y organizado lexicalmente, que interactúe de forma sencilla con un interlocutor colaborativo y que satisfaga necesidades inmediatas, como pedir cosas concretas, informarse sobre su ubicación, preguntar por un lugar, dar información personal o desenvolverse con cantidades, precios y horarios.

\section{Task-Based Learning (TBL)}

El enfoque de Willis busca un aprendizaje más centrado en el estudiante, el cual juega un papel más activo para descubrir y procesar el conocimiento, ya que el aprendiz es libre de usar las formas que necesita para dar un significado a lo que comunica y así completar la tarea. Doughty y Williams tratan de resolver la dicotomía entre dar prioridad a la comunicación o dar prioridad a la gramática y argumentan que enfocarse en la forma, no implica un enfoque tradicional, por el contrario, significa poner atención a los errores, para poder manejar adecuadamente la gramática que permitirá comunicarse, dominar estructuras problemáticas y usar estructuras secuenciadas e integradas para desarrollar el significado de una determinada función.

El desarrollo de tareas específicas con el idioma en las que se intercambia información, se usan métodos de ampliación y reformulación de la información son técnicas que constituyen esta metodología, con la cual se espera que los estudiantes procesen las formas, el significado y el uso al mismo tiempo.

\section{¿Qué es una tarea, (a task)?}

Lee define una tarea, a task, como:

Una actividad de clase o de ejercicio que tiene:

a) un objetivo alcanzable solamente por la interacción entre los participantes,

b) un mecanismo para la estructuración y secuenciación de la interacción,

c) un enfoque centrado en el intercambio de significados, y

d) un esfuerzo que requiere el aprendizaje de idiomas para comprender, manipular y/o producir la lengua meta por medio de un conjunto de planes de trabajo. (32)
Ellis presenta los siguientes puntos comunes entre varias definiciones dadas para una tarea y las define así: 1) una tarea es un plan de trabajo; 2) una tarea se enfoca principalmente en el significado; 3 ) una tarea involucra procesos reales de uso del lenguaje; 4) una tarea involucra procesos cognitivos; y 5) una tarea tiene un resultado comunicativo claramente definido.

Algunos ejemplos de tareas incluyen llenar un formulario, comprar un par de zapatos, hacer una reservación en una aerolínea, prestar un libro de la biblioteca, digitar una carta, preguntar por una dirección, etc. Este enfoque permite utilizar el idioma para situaciones de la vida real en el trabajo, la escuela, en casa, etc.

\section{Tipos de tareas}

Según Prabhu, existen esencialmente tres tipos de tareas, tasks: intercambio de información, information gap, en el que los estudiantes usan el lenguaje para compartir, dar y recibir alguna información; en la vida real se traduce en preguntas y respuestas como: ¿dónde queda el banco?, ¿de dónde eres?, el banco está en la próxima calle, soy americano. El segundo tipo de task son tareas de razonamiento, reasoning gap, en las que los estudiantes usan el lenguaje para compartir opiniones sobre determinadas situaciones comunicativas. El tercer tipo de tasks es problem-solving, en el que se usa el lenguaje para resolver problemas.

Por su parte, Willis afirma que existen seis tipos principales de tareas, que son: "listing, ordering and sorting, comparing, problem solving, sharing personal experiences and creative task" (29). Además puntualiza que las anteriores tareas difieren de un simple ejercicio de gramática, por cuanto se enfocan en el significado, meaning, y no en la forma, form, y conducen a un objetivo comunicativo específico. El proyecto incluirá los tipos de tareas, tasks, mencionados por los dos autores.

Willis expresa que cualquier tópico puede desarrollarse usando cualquier tipo de las seis tareas mencionadas, como se muestra en la figura 1.

Los tres primeros tipos de tareas son menos retadores para el estudiante, mientras que los tres últimos implican procesos de pensamiento más elevados; también el uso de la gramática es más exigente, ya que se utiliza en las habilidades productivas del idioma como son hablar, speaking, y escribir, writing. 


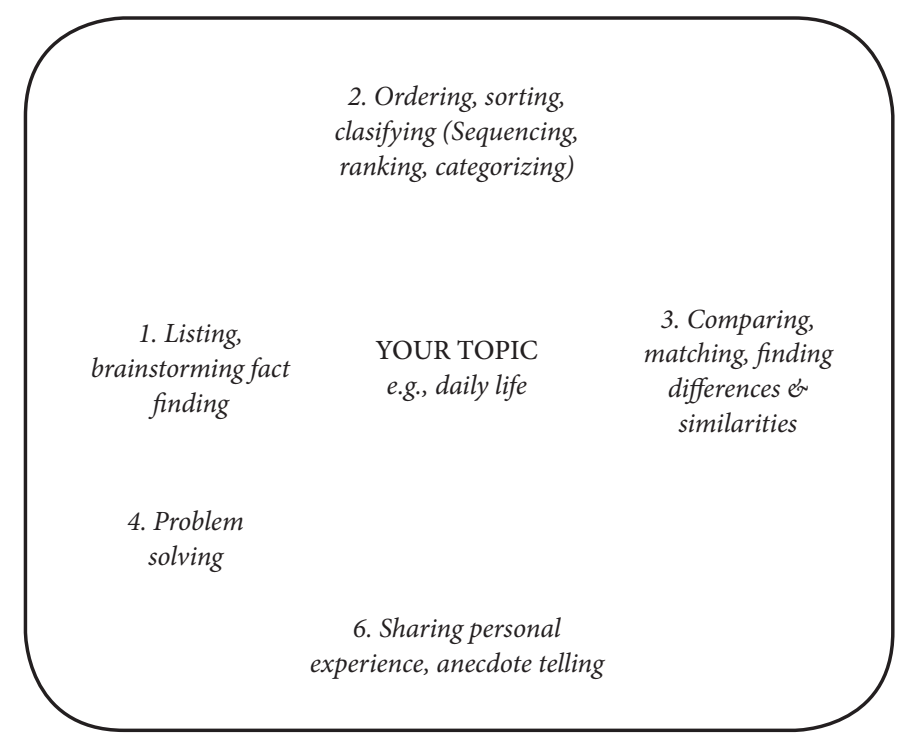

Figura 1. Typology for TBL Task Design

Fuente. Willis (29).

\section{Fases de una lección del TBL}

Las siguientes son las fases o componentes de la metodología propuesta por Willis para la planeación y desarrollo de una lección que contiene cuatro aspectos a tener en cuenta: la tarea previa, pre-task, el ciclo de la tarea, task, la focalización en el lenguaje, language focus y la fase después de la tarea, post-task. En el proyecto se trabajaron los anteriores tipos de tareas, combinándose con una metodología lúdica y teniendo en cuenta las fases del TBL, que se muestran en la tabla 1.

Tabla 1. Fases de una lección del твL

La tarea previa, pre-task - Introducción al tema y a la tarea

El docente introduce y define el tópico la vida diaria o daily life. También reactiva los conocimientos previos que el estudiante pueda tener sobre el tema (Schemata), usando preguntas motivadoras.

El docente usa actividades que ayuden a los estudiantes a recordar palabras y frases clave de utilidad (vocabulario) para dar al estudiante el input necesario, de modo que pueda desarrollar la tarea, task.

El docente se asegura de que los estudiantes entiendan las instrucciones para realizar la tarea, task, o puede presentar una grabación o un video con ejemplos de otras personas haciendo una actividad similar.

El docente presenta el procedimiento para realizar la tarea, task, (objetivos, procedimiento, tiempo, materiales, etc.).

El estudiante tiene en cuenta el nuevo vocabulario y las frases clave y se prepara para usarlos en el desarrollo de la tarea (task).

Actividades lúdicas: practicing, ordering, classifying, matchings, dragging, drilling, cloze, choosing the different words, checking, using crosswords, word search y otros.

II. La tarea, the task Tiene tres momentos:

La tarea: los estudiantes la realizan en parejas o en pequeños grupos, puede estar basada en la lectura o en la audición de un texto; mientras, el profesor monitorea la actividad y los estimula. Actividades propuestas:

Task 1. Hablar/escribir/preguntar sobre la rutina personal y de un compañero.

Conversación: daily life.

Actividades lúdicas: usar fotos personales, hacer videos y grabaciones, compartir videos y grabaciones con los compañeros, enviar correos, etc.
La planeación: los alumnos se preparan para reportar a sus compañeros de grupo cómo hicieron la tarea, lo que descubrieron o decidieron, es decir, los resultados. Ensayan lo que dirán o hacen un borrador de la versión escrita para leerla en el aula. El profesor se asegura que el propósito del reporte esté claro, actúa como consejero y los ayuda tanto en los trabajos orales como en los escritos. Los errores no son tenidos en cuenta en este momento.
El reporte: los estudiantes presentan los reportes a la clase. El profesor actúa como conductor, seleccionando a los participantes. De acuerdo con los logros obtenidos o no de la exposición, proporcionará la retroalimentación adecuada, con respecto a la forma y al contenido de la tarea; puede poner una grabación de otros estudiantes haciendo una actividad similar, para establecer comparaciones y luego hacer comentarios sobre los contenidos del reporte. 
(Viene)

\begin{tabular}{|c|c|}
\hline \multicolumn{2}{|c|}{ III: Focalización en el lenguaje, language focus o tratamiento de las estructuras gramaticales } \\
\hline $\begin{array}{l}\text { Análisis, analysis } \\
\text { Los estudiantes pueden escuchar grabaciones de tareas similares y com- } \\
\text { parar cómo lo hicieron. También pueden leer un texto similar a los que } \\
\text { ellos escribieron para realizar actividades de concientización e identificar } \\
\text { y procesar las estructuras específicas del texto o de la grabación y hacer } \\
\text { preguntas sobre lo que no hayan entendido. Los errores de los alumnos se } \\
\text { destacan y se crean actividades específicas para que los estudiantes puedan } \\
\text { practicar el uso de las formas lingüísticas correctas de una situación } \\
\text { comunicativa en particular. Este modelo requiere el uso de una forma } \\
\text { particular de gramática para llevar a cabo una tarea. Por ejemplo, si el } \\
\text { tema es una receta de comida, requerirá el uso de la forma imperativa, si } \\
\text { el tema es la decoración del aula de clase, se requerirán las preposiciones; } \\
\text { por tanto, este enfoque atiende al uso del lenguaje en situaciones reales. El } \\
\text { profesor repasará cada actividad de análisis de palabras, patrones o frases } \\
\text { útiles con los estudiantes, y puede seleccionar otros aspectos de la lengua a } \\
\text { partir de la etapa del reporte. } \\
\text { Actividades propuestas: ver videos de compañeros, leer textos de compa- } \\
\text { ñeros, intercambiar escritos, etc. }\end{array}$ & $\begin{array}{l}\text { Práctica, practice } \\
\text { El profesor conduce las actividades prácticas cuando sea necesario, } \\
\text { para que los alumnos se sientan confiados. Ellos ejercitan palabras, } \\
\text { frases y patrones de las actividades de análisis, y otras estructuras } \\
\text { que aparezcan en el texto de la tarea o en la etapa de reporte, además } \\
\text { escriben en sus libretas las unidades de la lengua que les resultan } \\
\text { útiles para la comunicación. }\end{array}$ \\
\hline \multicolumn{2}{|c|}{$\begin{array}{l}\text { IV. Después de la tarea, post-task } \\
\text { Retroalimentación y reflexión sobre la tarea: los estudiantes, a partir de la exposición de los resultados, se autoevalúan y reciben retroalimentación } \\
\text { de sus compañeros de aula y del profesor respecto del logro de los objetivos planteados, del logro de la tarea y del uso del lenguaje, de los recursos } \\
\text { utilizados, de las actividades, del tiempo, etc. A partir de la tarea realizada, pueden surgir otras tareas (tasks) dependientes. } \\
\text { Uso de rúbricas para evaluar el nivel de logro de la actividad comunicativa, el uso de la gramática y el desempeño de los estudiantes. Examen sobre } \\
\text { el tema estudiado. } \\
\text { Estándares del MCE (evaluación, can-do statements). }\end{array}$} \\
\hline
\end{tabular}

Fuente. Willis (72).

\section{El enfoque lúdico en el desarrollo de la competencia gramatical}

Para este proyecto no se tiene en cuenta el concepto de juego de manera tradicional. El concepto de lúdica va más allá y busca que el estudiante sea un participante activo que construye sus significados, por ello es que las diferentes actividades se planearán pensando en lo que el estudiante puede hacer, partiendo de pre-conceptos o presaberes que debe ir alcanzando progresivamente hasta llegar a conceptualizaciones y uso adecuado de la gramática. Esto es confirmado por Biggs, quien afirma que:

[...] el significado no se impone o se transmite en la instrucción directa, sino que se crea con las actividades de aprendizaje que el estudiante mismo realiza y la forma como se aproxima ellas. La metodología lúdica es importante en este proceso porque toma en cuenta las ideas y conceptos preconcebidos del estudiante y le permite avanzar y crear nuevas ideas para desarrollar su propia perspectiva y comprender otras alternativas y puntos de vista para lograr el aprendizaje. (13)

Aunque las actividades lúdicas pueden abarcar un amplio rango de posibilidades, en este proyecto se incluyen actividades en las que el estudiante tiene que hacer alguna actividad física para manipular y arrastrar información, escuchando, seleccionando, repitiendo información y además actividades mentales como completar, comparar, categorizar, clasificar información, etc., demostradas en las tasks y mini-tasks, en las que el estudiante debe hacer algo con el lenguaje. Esto también conduce al estudiante a desarrollar la observación y la reflexión sobre los procesos que realiza, para llegar a la abstracción y generalización de conceptos necesarios para el aprendizaje y desarrollo de la competencia gramatical, y aplicarla más adelante en situaciones de comunicación.

Las metodologías de aprendizaje lúdico son importantes por la práctica reflexiva y el razonamiento que hacen los estudiantes para llegar al conocimiento, los cuales se dan en el proceso acción-reflexión-conocimiento. Bolton describe que la práctica reflexiva y los enfoques lúdicos educativos requieren actividad y sensación, y que la experiencia sola no es suficiente para lograr el aprendizaje, por el contrario, debe haber una reflexión crítica que transforme la experiencia en aprendizaje. Según Bines y Watson, el proceso de reflexión crítica es inherente en el ciclo de aprendizaje:

Los métodos más apropiados para la enseñanza y el aprendizaje parecen ser los que tienen que ver con la investigación, el análisis, la experiencia y la resolución de 
problemas. Los enfoques lúdicos para el aprendizaje ayudan a generar interés, motivación, placer, compromiso y ayudan a estudiantes con diferentes estilos de aprendizaje a aproximarse a la asignatura desde diferentes perspectivas. Jugar con los conocimientos, temas y tópicos, implica un desafío para el estudiante, quien debe ir evaluando sus propios procesos de aprendizaje. Se apunta entonces hacia un enfoque en el que el aprendizaje es generado por la actividad del alumno, en lugar de lo indicado por el profesor. Esto cambió el papel de aprender de un modo pasivo hacia un proceso activo para el estudiante. (61)

Un aspecto importante para destacar es la evidencia de que las actividades lúdicas tienen un efecto positivo y mayor retención del conocimiento que la instrucción convencional, según Jonnavithula y Kinshuk. Sin embargo, es importante destacar que la motivación por sí sola no es suficiente para el aprendizaje, se deben crear estrategias que ayuden al estudiante a llevar a cabo un proceso de aprendizaje efectivo. Dada la riqueza de las actividades lúdicas en el aprendizaje, en este proyecto se incluyeron varias que se espera permitan el desarrollo de la competencia gramatical, rompiendo con el paradigma tradicional de la enseñanza y conduciendo al estudiante a un aprendizaje más agradable, efectivo y duradero.

\section{El uso de las TIC como recurso para favorecer el desarrollo de la competencia gramatical del inglés}

Yong presenta la importancia que se le está dando últimamente al uso de las tecnologías de la información y la comunicación, TIC, en el aprendizaje de los idiomas. Los investigadores y pedagogos están haciendo esfuerzos para descubrir mejores usos de la tecnología y mejorar el aprendizaje. Sumado a lo anterior, los pedagogos desean saber lo que funciona y no funciona al usar las TIC, para tomar decisiones más acertadas respecto al uso de la tecnología en los procesos de enseñanza. Ninguna de las dos inquietudes es fácil de contestar, debido a la gran variedad de herramientas tecnológicas y prácticas, desde el uso de Internet con videos, páginas web, chats, foros, etc., hasta conferencias interactivas. La autora especifica que:

[...] la tecnología implica más que el uso de un computador, se deben realizar actividades en las que el estudiante pueda practicar el idioma aprovechando la convergencia de los medios de comunicación. Para la práctica del idioma se emplean vídeos, programas de audio, la instrucción con ayuda de computadoras, Internet, comunicación mediada por ordenador, simulación, tecnologías del habla, procesamiento de textos, libros electrónicos, todo lo cual enriquece y mejora el aprendizaje por el acceso a materiales, oportunidades de comunicación, feedback y favorecimiento de la motivación en el aprendiz. El uso de las TIC es una gran ventaja para mejorar el aprendizaje del idioma. (12)

En el actual proyecto, Internet es una herramienta que permite el uso de gran variedad de materiales auditivos y visuales. También se utilizó la plataforma TEMA UNAB-Moodle para llevar un ambiente controlado de las actividades que se desarrollaron, y poder monitorear la participación y el progreso de cada estudiante. A su vez, el estudiante, a partir de los principios constructivistas, jugó un papel activo para su aprendizaje; el rol del docente cambió al de moderador, coordinador, facilitador y mediador del aprendizaje. Esta propuesta hizo uso de las TIC para presentar una perspectiva del proceso de enseñanza-aprendizaje del inglés a partir del enfoque comunicativo, que propone características muy especiales para el uso de los recursos.

\section{El método}

\section{Población y muestra}

La investigación se realizó en el Instituto de Lenguas de la Universidad Autónoma de Bucaramanga, unAB. La muestra tomó previamente la diagnóstica Placement Exam оОРт, que es una prueba internacional de Oxford que mide el nivel de inglés según los parámetros del MCE. Esta prueba es exigida por la UNAB como requisito de ingreso al Programa de inglés del Instituto de Lenguas. Según los resultados, la totalidad de los estudiantes de la muestra fueron ubicados en el nivel A1 del MCE.

Partiendo de este grupo homogéneo de competencia del inglés, se conformó una muestra aleatoria simple de 48 estudiantes de diferentes carreras universitarias, hombres y mujeres entre 16 y 25 años de edad, quienes realizan cursos de inglés en el periodo semestral. La muestra para la investigación fue poblacional total, dividiendo el grupo inicial en tres subgrupos: uno con 16 estudiantes, a los cuales se les aplicó el pretest como prueba piloto. Otro considerado el grupo experimental con 16 estudiantes, denominado para el presente estudio como $\mathrm{X}_{1}$; y el tercero, también con 16 estudiantes, denominado como $\mathrm{X}_{2}$, o grupo de control. 
El proceso que se llevó a cabo con la población seleccionada se muestra en la figura 2 .

\section{La hipótesis y las variables de la investigación}

En la tabla 2 se muestran la hipótesis y las variables de la investigación.

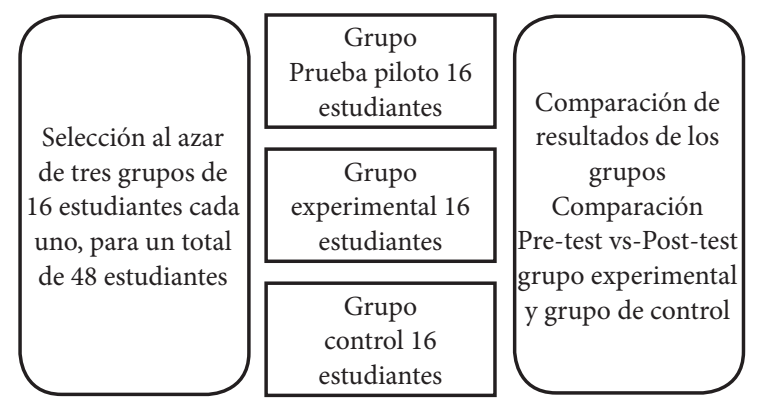

Figura 2. La muestra

Fuente. Elaboración propia.

Tabla 2. La hipótesis y las variables de la investigación

\begin{tabular}{|c|c|}
\hline \multicolumn{2}{|c|}{ Indicadores para medir la variable dependiente } \\
\hline \multirow{2}{*}{$\begin{array}{l}\text { Variable dependiente } \\
\text { (el efecto - lo que se mide) }\end{array}$} & Indicador 1. Manejo del vocabulario - Vida diaria. \\
\hline & $\begin{array}{l}\text { Indicador } 2 \text {. Usa oraciones con la estructura sujeto } I, \text { you, they, we+ } \\
\text { verbo+complemento, para expresar información sobre la vida diaria. }\end{array}$ \\
\hline \multirow{12}{*}{$\begin{array}{l}\text { El desarrollo de la compe- } \\
\text { tencia gramatical del tiempo } \\
\text { presente simple del inglés. } \\
\text { Conocimiento adquirido por } \\
\text { el estudiante. }\end{array}$} & Indicador 3. Usa oraciones con la estructura sujeto he, she, it+verbo \\
\hline & con s, es, o ies+complemento para expresar información sobre la vida diaria. \\
\hline & Indicador 4. Usa los sujetos $I$, you, they, we en oraciones con la \\
\hline & $\begin{array}{l}\text { estructura sujeto, auxiliar don't, verbo+complemento para expresar } \\
\text { oraciones sobre la vida diaria. }\end{array}$ \\
\hline & Indicador 5. Usa sujetos he, she, it, en oraciones con la estructura sujeto+ \\
\hline & $\begin{array}{l}\text { auxiliar doesn't,+verbo+complemento para expresar información sobre } \\
\text { la vida diaria. }\end{array}$ \\
\hline & $\begin{array}{l}\text { Indicador } 6 \text {. Hace preguntas sobre la vida diaria usando la estructura: auxiliar } \\
\text { do+sujeto+verbo+complemento. }\end{array}$ \\
\hline & $\begin{array}{l}\text { Indicador } 7 \text {. Hace preguntas sobre la vida diaria usando la estructura: auxiliar } \\
\text { does+sujeto+verbo+complemento. }\end{array}$ \\
\hline & $\begin{array}{l}\text { Indicador } 8 \text {. Hace preguntas sobre la vida diaria utilizando la estructura: } W h+\text { Aux } \\
\text { do+sujeto+verbo+complemento. }\end{array}$ \\
\hline & $\begin{array}{l}\text { Indicador } 9 \text {. Hace preguntas sobre la vida diaria utilizando la estructura } \\
\text { Wh+Aux does+sujeto+verbo+complemento. }\end{array}$ \\
\hline & Indicador 10. Objetivo gramatical del nivel A1 - MCE. \\
\hline & $\begin{array}{l}\text { Usa el tiempo presente simple en oraciones sencillas relacionadas con los } \\
\text { temas de la vida diaria, daily life (Se obtiene del análisis de los nueve indicadores anteriores). }\end{array}$ \\
\hline Variable independiente & Indicadores para manipular la variable independiente. \\
\hline \multirow[t]{5}{*}{$\begin{array}{l}\text { Variable independiente } \\
\text { (causa) }\end{array}$} & $\begin{array}{l}\text { Indicador } 1 \text { de la metodología lúdica: participación activa del estudiante en el proceso de } \\
\text { aprendizaje. }\end{array}$ \\
\hline & Indicador 2 de la metodología lúdica: práctica y entrenamiento. \\
\hline & Indicador 3 de la metodología lúdica: preconceptos para superar las dificultades. \\
\hline & Indicador 4 de la metodología lúdica: aprender haciendo. \\
\hline & Indicador 5 de la metodología lúdica: actividad mental y reflexiva. \\
\hline \multirow{4}{*}{$\begin{array}{l}\text { La metodología lúdica } \\
\text { (influencia de la metodología } \\
\text { en el desarrollo de la compe- } \\
\text { tencia gramatical) }\end{array}$} & Indicador 6 de la metodología lúdica: resolución de problemas. \\
\hline & Indicador 7 de la metodología lúdica: evaluación de desempeño y logros \\
\hline & Indicador 8. Creación de actividades de aprendizaje. \\
\hline & Indicador 9. Metodología fácil y amena. \\
\hline
\end{tabular}

Fuente. Elaboración propia. 


\section{Los instrumentos y su justificación}

En la tabla 3 se muestran los instrumentos utilizados, con su respectivo propósito, en la presente investigación:

Tabla 3. Instrumentos de la propuesta de investigación

\begin{tabular}{|c|c|c|c|}
\hline Instrumentos & Cuándo & Quiénes & ¿Para qué? \\
\hline $\begin{array}{l}\text { Primer instrumento. Prueba de } \\
\text { entrada sobre la gramática del } \\
\text { tiempo presente simple, respecto } \\
\text { al tema daily life. } \\
\qquad \downarrow \\
\text { Prueba de entrada o pretest }\end{array}$ & $\begin{array}{l}\text { Antes de la interven- } \\
\text { ción con la metodolo- } \\
\text { gía lúdica. }\end{array}$ & $\begin{array}{l}\text { Grupo piloto, grupo } \\
\text { experimental y gru- } \\
\text { po de control. }\end{array}$ & $\begin{array}{l}\text { Pretest para definir el nivel de desarrollo de la competen- } \\
\text { cia gramatical del tiempo presente simple, antes de iniciar } \\
\text { la intervención con la metodología lúdica. Los resultados } \\
\text { obtenidos se compararán con la prueba de salida para } \\
\text { verificar el éxito o el fracaso de la propuesta metodológica. }\end{array}$ \\
\hline $\begin{array}{l}\text { Segundo instrumento. Prueba } \\
\text { de salida sobre la gramática del } \\
\text { tiempo presente simple, respecto } \\
\text { al tema daily life. } \\
\qquad \downarrow \\
\text { Prueba de salida o postest }\end{array}$ & $\begin{array}{l}\text { Después de finalizar las } \\
10 \text { horas de interven- } \\
\text { ción, en la hora once. }\end{array}$ & $\begin{array}{l}\text { Grupo experimental } \\
\text { y grupo de control. }\end{array}$ & $\begin{array}{l}\text { El postest se comparará con el pretest, para definir el nivel } \\
\text { de desarrollo de la competencia gramatical del tiempo pre- } \\
\text { sente simple después de la intervención de la metodología } \\
\text { lúdica aplicada al grupo experimental. Al grupo de control } \\
\text { no se le hará ninguna intervención metodológica. }\end{array}$ \\
\hline $\begin{array}{l}\text { Tercer instrumento. } \\
\text { Cuestionario entregado a estu- } \\
\text { diantes que fueron intervenidos } \\
\text { con la metodología lúdica. } \\
\downarrow \\
\text { Cuestionario cerrado: Escala de } \\
\text { Likert }\end{array}$ & $\begin{array}{l}\text { Después de finalizar las } \\
10 \text { horas de interven- } \\
\text { ción y de haber aplica- } \\
\text { do la prueba de salida, } \\
\text { en la hora doce. }\end{array}$ & Grupo experimental & $\begin{array}{l}\text { A los estudiantes el cuestionario pretende medirles el } \\
\text { grado de aceptación de la metodología lúdica. }\end{array}$ \\
\hline
\end{tabular}

Fuente. Elaboración propia.

\section{Planteamiento del diseño para un contraste de medias}

Para la presente investigación se utilizó el siguiente diseño propuesto por Valenzuela y Flores, como se muestra en la figura 3:

$$
\begin{array}{llll}
\text { Grupo Experimental } & \mathrm{O}_{1} & \mathrm{X} & \mathrm{O}_{2} \\
\text { Grupo de Control } & \mathrm{O}_{1} & & \mathrm{O}_{2}
\end{array}
$$

Figura 3. Diseño de un contraste de medias pre y postest Fuente. Valenzuela y Flores (88).

$\mathrm{O} 1=$ Pretest $=$ Precede siempre al tratamiento de los sujetos con la metodología lúdica. Total de nueve indicadores, cada uno con cuatro respuestas para un total de 36 respuestas, cada una con valor de 1 .
$\mathrm{X}=$ Metodología lúdica $=$ La variable independiente que el investigador manipula. Total de 8 indicadores. Cada uno con una sola posibilidad de respuesta=Escala de Likert 20\%, 40\%, 60\%, 80\%, 100\%.

$\mathrm{O} 2=$ Postest $=$ Posterior a $\mathrm{X}=$ a variable dependiente $=$ Lo que se desea comprobar=el nivel de desarrollo de la gramática. Total de nueve indicadores, cada uno con cuatro respuestas, para un total de 36 respuestas, cada una con valor de 1 .

\section{Resultados}

\section{Análisis de medias - Grupo experimental postest}

Para el análisis de medias se tomaron los siguientes valores obtenidos en el grupo experimental para el postest:

\begin{tabular}{|l|l|l|l|l|l|l|l|l|l|l|}
\hline $\begin{array}{l}\text { Grupo } \\
\text { experimental } \\
\text { postest }\end{array}$ & Media & I1 & I2 & I3 & I4 & I5 & I6 & I7 & I8 & I9 \\
\cline { 2 - 10 } & 3,781 & 3,688 & 3,375 & 3,375 & 3,438 & 3,000 & 3,313 & 3,438 & 3,063 \\
\hline
\end{tabular}


Los resultados que se obtuvieron después que el grupo recibió el tratamiento, cambiaron de manera positiva y todos los valores medios se encuentran por encima de 3,1, como se muestra en la figura 4:

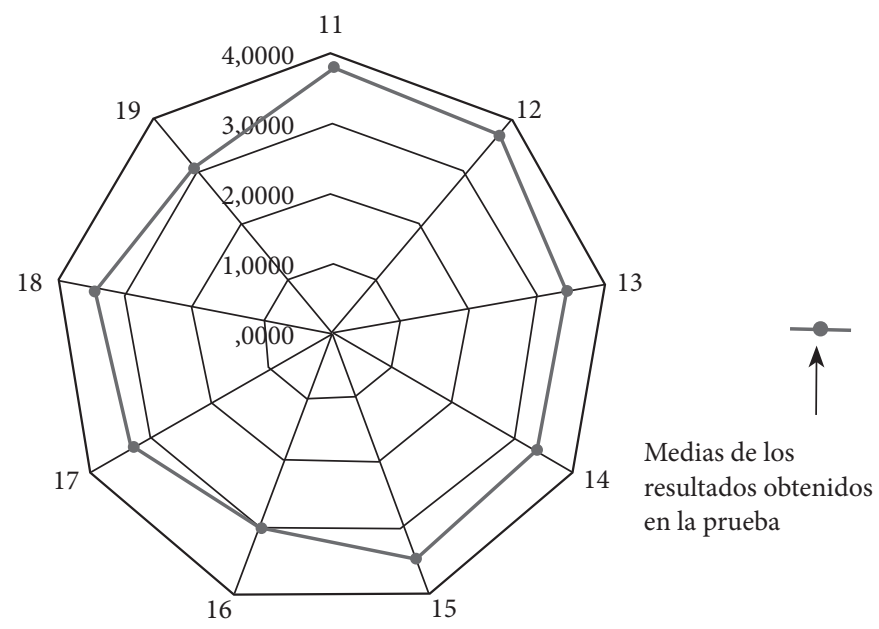

Figura 4. Análisis de medias - Grupo experimental postest Fuente. Datos estudiantes de pregrado nivel A1 del programa de inglés. UNAB, 2013.

\section{Análisis de medias. Diferencia entre calificaciones del postest para los grupos experimental y control}

Para la diferencia entre calificaciones en el postest, para los grupos experimental y de control se tomaron los siguientes valores obtenidos:

\begin{tabular}{|l|c|c|c|c|c|c|c|c|c|}
\hline \multicolumn{1}{|c|}{ Medias } & I1 & I2 & I3 & I4 & I5 & I6 & I7 & I8 & I9 \\
\hline $\begin{array}{l}\text { Diferencias } \\
\text { entre las } \\
\text { evaluaciones }\end{array}$ & 2,13 & 0,75 & 2,13 & 2,31 & 2,06 & 2,00 & 2,69 & 3,00 & 2,69 \\
\hline
\end{tabular}

En todos los casos, la diferencia fue mayor; el grupo experimental obtuvo resultados significativos. La diferencia entre las calificaciones obtenidas en los resultados del postest para los grupos experimental y de control se observa en la figura 5:

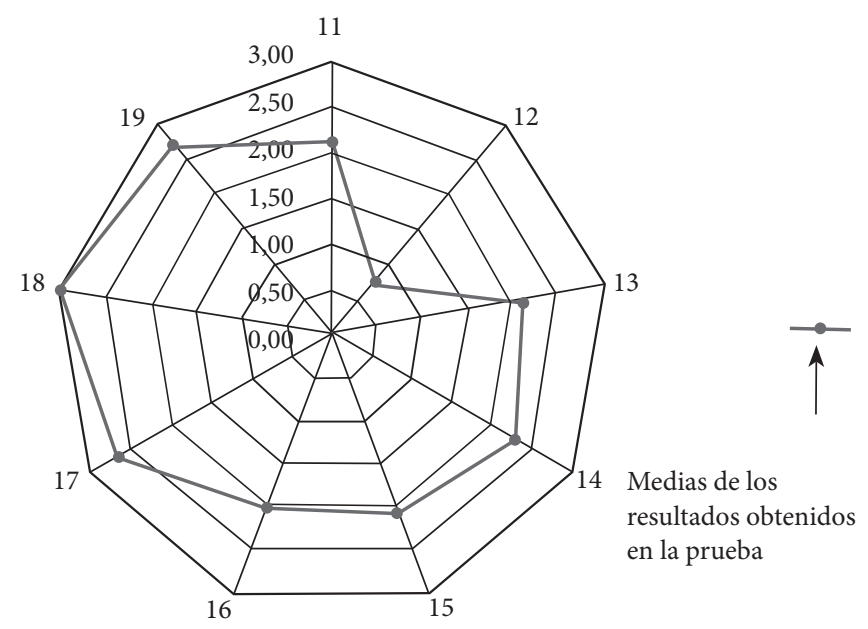

Figura 5. Diferencias entre calificaciones del postest para los grupos experimental y de control Fuente. Datos estudiantes de pregrado nivel A1 del programa de inglés. UNAB, 2013. 


\section{Prueba de hipótesis}

La tabla 4 muestra los resultados respecto a la prueba de hipótesis de las diferencias entre las calificaciones de los estudiantes que estaban en el grupo experimental y los del grupo control. Se presentan en las columnas la prueba de Levene para determinar si se cumple el su- puesto de homocedasticidad, el nivel de significancia, la prueba $t$-student y su respectivo nivel de significancia. Se puede concluir que en todos los casos existen diferencias significativas, lo que permite concluir que el tratamiento aplicado con la metodología lúdica fue significativo de manera positiva en el proceso de enseñanza.

Tabla 4. Prueba de hipótesis para la comparación entre los grupos experimental y de control para el postest

\begin{tabular}{|c|c|c|c|c|c|c|c|c|c|c|}
\hline \multicolumn{11}{|c|}{ Prueba de muestras independientes } \\
\hline & & \multicolumn{2}{|c|}{$\begin{array}{c}\text { Prueba de Levene para la } \\
\text { igualdad de varianzas }\end{array}$} & \multicolumn{7}{|c|}{ Prueba T para la igualdad de medias } \\
\hline & & \multirow[b]{2}{*}{$\mathrm{F}$} & \multirow[b]{2}{*}{ Sig. } & \multirow[b]{2}{*}{$\mathrm{t}$} & \multirow[b]{2}{*}{ gl } & \multirow[b]{2}{*}{$\begin{array}{c}\text { Sig. } \\
\text { (bilateral) }\end{array}$} & \multirow[b]{2}{*}{$\begin{array}{l}\text { Diferencia } \\
\text { de medias }\end{array}$} & \multirow[b]{2}{*}{$\begin{array}{l}\text { Error típ. de } \\
\text { la diferencia }\end{array}$} & \multicolumn{2}{|c|}{$\begin{array}{l}95 \% \text { Intervalo de } \\
\text { confianza para la }\end{array}$} \\
\hline & & & & & & & & & Inferior & Superior \\
\hline \multirow[t]{2}{*}{11} & $\begin{array}{l}\text { Se han asumido varianzas } \\
\text { iguales }\end{array}$ & 20,18 & 0,00 & 8,14 & 30,00 & 0,00 & 2,13 & 0,26 & 1,59 & 2,66 \\
\hline & $\begin{array}{l}\text { No se han asumido } \\
\text { varianzas iguales }\end{array}$ & & & 8,14 & 19,07 & 0,00 & 2,13 & 0,26 & 1,58 & 2,67 \\
\hline \multirow[t]{2}{*}{12} & $\begin{array}{l}\text { Se han asumido varianzas } \\
\text { iguales }\end{array}$ & 1,21 & 0,28 & 2,57 & 30,00 & 0,02 & 0,75 & 0,29 & 0,15 & 1,35 \\
\hline & $\begin{array}{l}\text { No se han asumido } \\
\text { varianzas iguales }\end{array}$ & & & 2,57 & 27,96 & 0,02 & 0,75 & 0,29 & 0,15 & 1,35 \\
\hline \multirow[t]{2}{*}{13} & $\begin{array}{l}\text { Se han asumido varianzas } \\
\text { iguales }\end{array}$ & 1,02 & 0,32 & 4,81 & 30,00 & 0,00 & 2,13 & 0,44 & 1,22 & 3,03 \\
\hline & $\begin{array}{l}\text { No se han asumido } \\
\text { varianzas iguales }\end{array}$ & & & 4,81 & 29,30 & 0,00 & 2,13 & 0,44 & 1,22 & 3,03 \\
\hline \multirow[t]{2}{*}{14} & $\begin{array}{l}\text { Se han asumido varianzas } \\
\text { iguales }\end{array}$ & 2,37 & 0,13 & 5,13 & 30,00 & 0,00 & 2,31 & 0,45 & 1,39 & 3,23 \\
\hline & $\begin{array}{l}\text { No se han asumido } \\
\text { varianzas iguales }\end{array}$ & & & 5,13 & 25,22 & 0,00 & 2,31 & 0,45 & 1,39 & 3,24 \\
\hline
\end{tabular}

\begin{tabular}{|c|c|c|c|c|c|c|c|c|c|c|}
\hline \multicolumn{11}{|c|}{ Prueba de muestras independientes } \\
\hline & & \multicolumn{2}{|c|}{$\begin{array}{c}\text { Prueba de Levene para la } \\
\text { igualdad de varianzas }\end{array}$} & \multicolumn{7}{|c|}{ Prueba T para la igualdad de medias } \\
\hline & & \multirow[b]{2}{*}{$\mathrm{F}$} & \multirow[b]{2}{*}{ Sig. } & \multirow[b]{2}{*}{$\mathrm{t}$} & \multirow[b]{2}{*}{$\mathrm{gl}$} & \multirow[b]{2}{*}{$\begin{array}{l}\text { Sig. } \\
\text { (bilateral) }\end{array}$} & \multirow[b]{2}{*}{$\begin{array}{l}\text { Diferencia } \\
\text { de medias }\end{array}$} & \multirow[b]{2}{*}{$\begin{array}{l}\text { Error típ. de } \\
\text { la diferencia }\end{array}$} & \multicolumn{2}{|c|}{$\begin{array}{l}95 \% \text { Intervalo de } \\
\text { confianza para la }\end{array}$} \\
\hline & & & & & & & & & Inferior & Superior \\
\hline \multirow[t]{2}{*}{15} & $\begin{array}{l}\text { Se han asumido varianzas } \\
\text { iguales }\end{array}$ & 17,09 & 0,00 & 4,14 & 30,00 & 0,00 & 2,06 & 0,50 & 1,04 & 3,08 \\
\hline & $\begin{array}{l}\text { No se han asumido } \\
\text { varianzas iguales }\end{array}$ & & & 4,14 & 22,06 & 0,00 & 2,06 & 0,50 & 1,03 & 3,10 \\
\hline \multirow[t]{2}{*}{16} & $\begin{array}{l}\text { Se han asumido varianzas } \\
\text { iguales }\end{array}$ & 2,65 & 0,11 & 4,78 & 30,00 & 0,00 & 2,00 & 0,42 & 1,15 & 2,85 \\
\hline & $\begin{array}{l}\text { No se han asumido } \\
\text { varianzas iguales }\end{array}$ & & & 4,78 & 27,00 & 0,00 & 2,00 & 0,42 & 1,14 & 2,86 \\
\hline \multirow[t]{2}{*}{17} & $\begin{array}{l}\text { Se han asumido varianzas } \\
\text { iguales }\end{array}$ & 2,86 & 0,10 & 8,65 & 30,00 & 0,00 & 2,69 & 0,31 & 2,05 & 3,32 \\
\hline & $\begin{array}{l}\text { No se han asumido } \\
\text { varianzas iguales }\end{array}$ & & & 8,65 & 26,58 & 0,00 & 2,69 & 0,31 & 2,05 & 3,33 \\
\hline \multirow[t]{2}{*}{18} & $\begin{array}{l}\text { Se han asumido varianzas } \\
\text { iguales }\end{array}$ & 0,21 & 0,65 & 7,36 & 30,00 & 0,00 & 3,00 & 0,41 & 2,17 & 3,83 \\
\hline & $\begin{array}{l}\text { No se han asumido } \\
\text { varianzas iguales }\end{array}$ & & & 7,36 & 29,70 & 0,00 & 3,00 & 0,41 & 2,17 & 3,83 \\
\hline
\end{tabular}

Prueba de muestras independientes

\begin{tabular}{|c|c|c|c|c|c|c|c|c|c|c|}
\hline & & \multicolumn{2}{|c|}{\begin{tabular}{|c|}
$\begin{array}{c}\text { Prueba de Levene para la } \\
\text { igualdad de varianzas }\end{array}$ \\
\end{tabular}} & \multicolumn{7}{|c|}{ Prueba T para la igualdad de medias } \\
\hline & & \multirow[t]{2}{*}{$\begin{array}{ll} \\
\end{array}$} & \multirow[b]{2}{*}{ Sig. } & \multirow[b]{2}{*}{$\mathrm{t}$} & \multirow[b]{2}{*}{$\mathrm{gl}$} & \multirow[b]{2}{*}{$\begin{array}{c}\text { Sig. } \\
\text { (bilateral) }\end{array}$} & \multirow[b]{2}{*}{$\begin{array}{l}\text { Diferencia } \\
\text { de medias }\end{array}$} & \multirow[b]{2}{*}{$\begin{array}{l}\text { Error típ. de } \\
\text { la diferencia }\end{array}$} & \multicolumn{2}{|c|}{$\begin{array}{l}95 \% \text { Intervalo de } \\
\text { confianza para la }\end{array}$} \\
\hline & & & & & & & & & Inferior & Superior \\
\hline \multirow[t]{2}{*}{19} & $\begin{array}{l}\text { Se han asumido varianzas } \\
\text { iguales }\end{array}$ & 0,23 & 0,64 & 7,69 & 29,00 & 0,00 & 2,73 & 0,35 & 2,00 & 3,45 \\
\hline & $\begin{array}{l}\text { No se han asumido } \\
\text { varianzas iguales }\end{array}$ & & & 7,66 & 28,04 & 0,00 & 2,73 & 0,36 & 2,00 & 3,46 \\
\hline \multirow[t]{2}{*}{ Gramatica } & $\begin{array}{l}\text { Se han asumido varianzas } \\
\text { iguales }\end{array}$ & 6,01 & 0,02 & 7,58 & 30,00 & 0,00 & 17,56 & 2,32 & 12,83 & 22,30 \\
\hline & $\begin{array}{l}\text { No se han asumido } \\
\text { varianzas iguales }\end{array}$ & & & 7,58 & 22,43 & 0,00 & 17,56 & 2,32 & 12,76 & 22,36 \\
\hline \multirow{2}{*}{$\begin{array}{l}\text { Gramatica_vocabul } \\
\text { ario }\end{array}$} & $\begin{array}{l}\text { Se han asumido varianzas } \\
\text { iguales }\end{array}$ & 6,50 & 0,02 & 7,94 & 30,00 & 0,00 & 19,56 & 2,46 & 14,53 & 24,59 \\
\hline & $\begin{array}{l}\text { No se han asumido } \\
\text { varianzas iguales }\end{array}$ & & & 7,94 & 22,12 & 0,00 & 19,56 & 2,46 & 14,46 & 24,67 \\
\hline
\end{tabular}

Fuente. Datos estudiantes de pregrado nivel A1 del programa de inglés. UnAB, 2013. 


\section{Análisis de datos de la prueba cuestionario cerrado}

Como se mencionó anteriormente, el instrumento tres o cuestionario cerrado buscaba medir la variable independiente (Causa). Se intentaba probar la hipótesis de si la metodología lúdica favorecía o no el desarrollo de la competencia gramatical del tiempo presente simple, y para ello se plantearon como fundamento ocho indicadores propuestos en la operacionalización de las varia- bles. Para mediar las variables se tuvo en cuenta la Escala de Likert, la cual pretendía medir el grado de aceptación de los estudiantes respecto de la metodología lúdica.

\section{Método de mitades - Análisis de fiabilidad cuestionario cerrado}

La tabla 5 presenta el estadístico de fiabilidad, en el que la muestra se ha repartido en dos mitades.

Tabla 5. Estadístico de fiabilidad - Muestra en dos mitades

\begin{tabular}{|l|l|l|l|}
\hline Alfa de Cronbach & Parte 1- Indicadores 1-4 & Valor &, 930 \\
\hline & & N de elementos & $4^{\mathrm{a}}$ \\
\hline & Parte 2 & Valor &, 742 \\
\hline & Indicadores 5-8- & N de elementos & $4^{\mathrm{b}}$ \\
\hline & N total de elementos & 8 \\
\hline Correlación entre formas & \multicolumn{2}{|l|}{, 870} \\
\hline $\begin{array}{l}\text { Coeficiente de } \\
\text { Spearman-Brown }\end{array}$ & Longitud igual &, 930 \\
\hline & Longitud desigual &, 930 \\
\hline Dos mitades de Guttman & \multicolumn{2}{|l|}{, 929} \\
\hline
\end{tabular}

Fuente. Datos estudiantes de pregrado nivel A1 del programa de inglés. UNAB, 2013.

En los resultados se observa:

Para la parte 1. Hay un valor de Alfa de Cronbach $=$ 0,930 , en el que se han integrado los primeros cuatro indicadores de la metodología lúdica. Dado que en este instrumento de medición oscila de 0 a 1 , cuanto más se aproxime a su valor máximo, 1 , mayor es la fiabilidad de la escala. Entonces, para la parte 1 hay un valor muy cercano a 1 que es $=0,930$, indicando que hay fiabilidad en la escala mayor.

Para la parte 2. Hay un valor de Alfa de Cronbach $=0,742$, integrado por cuatro indicadores de cinco al ocho de la metodología lúdica. Se tiene en cuenta que este instrumento oscila entre 0 y 1 , pero se considera que los valores de Alfa superiores a 0,7 o 0,8 son suficientes para garantizar la fiabilidad de la escala. Se puede predecir que para la parte dos, el valor de Alfa de Cronbach $=0,742$; este valor resultante es suficiente también para garantizar la fiabilidad como buena.

En el anterior cuadro de diálogo se encuentra otro dato estadístico de fiabilidad, que es la correlación entre formas con un valor $=0,870$. Por ser un valor cercano a 1 , se puede predecir que hay una correlación alta, ya que la correlación en su escala de medición se encuentra en un intervalo de - 1 a 1 , siendo el valor de 1 una correlación excelente. Por ser el Coeficiente de Spearman una escala de medición o correlación entre dos variables, se puede asociar en los resultados de la fiabilidad obtenida entre las dos partes. Es así como el Coeficiente de Spearman $=0,930$ permite inferir que hay un alto grado de fiabilidad en las escalas de medición.

\section{Prueba de la hipótesis de cuestionario cerrado}

Para probar esta hipótesis se toma como referencia el valor fiabilidad de la muestra, respecto al Coeficiente de Spearman, el cual se encuentra en un valor bastante cercano a 1; en la tabla de resultados de Estadísticos de Fiabilidad se dio un valor de 0,930 , determinando el valor como muy bueno. Con base en este referente se puede concluir que la metodología lúdica favorece el desarrollo de la gramática del presente simple del inglés del nivel A1, aceptándose la hipótesis planteada.

\section{Discusión de resultados y conclusiones}

Los hallazgos del presente estudio verifican los supuestos teóricos sobre la importancia de la metodología lúdica para la enseñanza de la gramática; Meyer y Land describen que este proceso de aprendizaje también implica perder algunas preconcepciones personales, para ganar un nuevo 
punto de vista del mundo. Aunque las actividades lúdicas pueden abarcar un amplio rango de posibilidades, en este proyecto se incluyen actividades en las que el estudiante tiene que hacer alguna actividad física para manipular y arrastrar información, escuchando, seleccionando, repitiendo información y además realizar actividades mentales como completar, comparar, categorizar, clasificar información, etc., demostradas en las tasks y mini-tasks, en las que el estudiante debe hacer algo con el lenguaje.

Según los resultados de la prueba de Levene y de la prueba t-student, respecto de la prueba de la hipótesis de las diferencias entre las calificaciones de los estudiantes que estaban ubicados en los grupos experimental y de control, para el pretest/postest, arrojaron un nivel de significancia en el que se puede concluir que en todos los casos existen diferencias significativas, como se observa en la figura 6, en el que se muestra el progreso obtenido en las calificaciones:

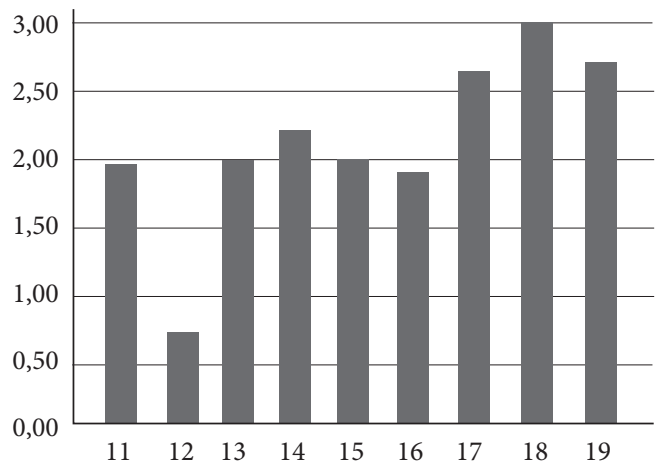

Figura 6. Diferencia entre las calificaciones por indicadores de los grupos de control y experimental en el postest

Fuente. Datos estudiantes de pregrado nivel A1 del programa de inglés. UNAB, 2013.

Para todos los indicadores la diferencia fue mayor; el grupo experimental obtuvo resultados significativos, lo que permite concluir que el tratamiento aplicado con la metodología lúdica fue significativo y de manera positiva en el proceso de enseñanza, aceptándose así la hipótesis del presente estudio. Los resultados positivos obtenidos, presumiblemente, se deben a las características especiales que se tuvieron en cuenta para su diseño: a) el énfasis en el diseño de los materiales, b) el trabajo fuerte en el vocabulario, c) el tipo de estrategias de lectura aplicadas teniendo en cuenta la metodología lúdica y el TBL, d) el rol del docente como diseñador y docente del curso, y f) el interés de los estudiantes y su participación activa mediante el uso de las TIC para su aprendizaje.

Otro hallazgo importante en el estudio fue la positiva aceptación de los estudiantes de la variable in- dependiente (Causa-metodología lúdica), en la que se tomó como referencia el valor de fiabilidad de la muestra, respecto del Coeficiente de Spearman, que arrojó un valor bastante cercano a 1 : se dio un valor de 0,930 , determinando el valor como muy bueno.

\section{Referencias}

Biggs, John. Teaching for Quality Learning at University. Buckingham: Open University Press, 1999. Impreso.

Bines, Hazel y Watson, David. Developing Professional Education. Buckingham: Open University Press, 1992. Impreso.

Bolton, Gillie. Reflective Practice: Writing and Professional Development. London: Sage Publications, 2005. Impreso.

Common European Framework of Reference for Languages. Common European Framework of Reference for Languages: Learning Teaching Assessment. Cambridge, England: Cambridge University Press, 2004. Impreso.

Doughty, Catherine y Williams, Jessica. Pedagogical Choices in Focus on Form. Cambridge, England: Cambridge University Press, (1999). Impreso.

Ellis, Rod. Task-Based Language Learning and Teaching. Oxford: Oxford University Press, 2003. Impreso.

Jonnavithula, Lalitha y Kinshuk, Dinn. "Exploring multimedia educational games: An aid to reinforce classroom teaching and learning." Conference Paper Fourth IASTED International Conference on Web-Based Education. Calgary, Canada: Acta Press, 2005. Impreso.

Lee, James. Tasks and Communicating in Language Classrooms. New York, EE.UU.: McGraw-Hill, 2000. Impreso.

Meyer, Erik y Land, Ray. "Implications of threshold concepts for course design and evaluation". En Meyer J.H.F. y Land R. (eds.), Overcoming Barriers to Student Understanding: threshold concepts and troublesome knowledge. Londres y Nueva York: Routledge, 2006. Impreso.

Oxford University Press. оopt Placement Test. 12 abr. 2013. Web. http://www.italki.com/p/oet.htm

Prabhu, Neiman Stern. Second Language Pedagogy. Oxford, UK: Oxford University Press, 1987. Impreso.

Valenzuela, Ricardo y Flores, Manuel. Fundamentos de investigación educativa. Editorial Digital del Tecnológico de Monterrey. México. Primera edición, 2012. Impreso.

Willis, Jane. A framework for task-based learning. Harlow, UK: Addison Wesley Longman, 1996. Impreso.

Yong, Zhao. "Recent Developments in Technology and Language Learning: A Literature Review and Meta-analysis." Michigan State University. ProQuest Education Journals 21.1 (2003): 1-7. 10 mar. 2013. Web. http:// search.proquest.com.bases.unab.edu.co/docview/750 477643/13CFA4B8169369F8B69/18?accountid=41597 